\title{
AN APPROXIMATION THEOREM FOR A CLASS OF OPERATORS ${ }^{1}$
}

\author{
G. K. LEAF
}

It is well known that if $U$ is a unitary operator in a Hilbert space $H$, then the following approximation theorem is an immediate consequence of the spectral representation for the operator $U$.

Theorem A. (a) Let $\left\{E_{t}: 0 \leqq t \leqq 2 \pi\right\}$ denote the family of spectral projections associated with $U$; then if $\epsilon>0$ and $\alpha, 0 \leqq \alpha \leqq 2 \pi$, are given and if $x$ is any element in the range of the projection $E_{\alpha+\cdot}-E_{\alpha}$, we have:

$$
\left\|\left(U-e^{i \alpha}\right) x\right\| \leqq \epsilon\|x\|
$$

(b) moreover, for this same $\epsilon$, there exists a finite collection of closed linear manifolds in $H$ such that $H$ is the direct sum of these manifolds; and in each subspace, $U$ behaves as in part (a).

E. R. Lorch [7] extended this theorem to certain classes of operators in a reflexive Banach space. The class considered by Lorch consists of those bounded, invertible operators $V$ for which the norms of their iterates are restricted by the condition, $\left\|V^{n}\right\|=O(1)$ as $|n|$ tends to infinity.

The author [6] extended the results or Lorch to a somewhat larger class restricted by the condition, $\left\|V^{n}\right\|=O(|n|)$. This extension was made through the use of methods developed by N. Dunford [4], [5].

In the present paper, the result is extended to a much larger class of operators by using the methods of Harmonic analysis as developed by A. Beurling [1], J. Wermer [8], Y. Domar [3], and others. It should be mentioned that this extension might have been possible through the use of methods developed by F. Wolf [9] in his spectral theory for operators based on the generalized trigonometric integrals of $\mathrm{S}$. Bochner. The present class is restricted by the condition,

(i) $\left\|V^{n}\right\|=O\left(|n|^{a}\right)$ as $|n|$ tends to infinity, for some $q>0$.

An operator $V$, defined in a Banach space $B$, which satisfies condition (i) is easily seen to have it spectrum on the circumference of the unit circle. Moreover, the usual operational calculus may be extended by introducing a certain weighted algebra associated with the sequence $\left\{\left\|V^{n}\right\|: n=0, \pm 1, \cdots\right\}$. Such algebras were introduced by Beurling [1], and later generalized by Wermer and Domar.

Received by the editors August 13, 1964.

1 Work performed under the auspices of the U. S. Atomic Energy Commission. 
Definition 1. Let $\rho_{n}$ and $d_{n}, n=0, \pm 1, \cdots$ be sequences of positive numbers such that $\rho_{n} \geqq 1, \rho_{n+m} \leqq \rho_{n} \rho_{m}$, and $\rho_{n} \leqq d_{n}$, where $d_{n}$ is an even, increasing sequence. Denote by $A_{\rho}$ the space of all continuous, periodic functions $f(\theta)$ whose Fourier coefficients $f_{n}$ satisfy $\sum_{n=-\infty}^{\infty} \rho_{n}\left|f_{n}\right|<\infty$.

When given the norm $\|f\|=\sum_{-\infty}^{\infty} \rho_{n}\left|f_{n}\right|$, the space $A_{\rho}$ becomes a commutative Banach algebra under pointwise multiplication Moreover, if $\rho_{n}$ is further restricted by $\sum_{-\infty}^{\infty} \log \rho_{n} /\left(1+n^{2}\right)<\infty$, the algebra $A_{\rho}$ is regular. In the present application, we take $\rho_{n}=\left\|V^{n}\right\|$; the relevant conditions satisfied by $\rho_{n}$ follow directly from the restriction of polynomial growth on $\left\|V^{n}\right\|$.

Using the algebra $A_{\rho}$, an operational calculus may be generated for $V$ by setting $f(V)=\sum_{-\infty}^{\infty} f_{n} V^{n}$, for such $f$ in $A_{\rho}$. In order to apply the methods of harmonic analysis, we shall drop down to the space $B$ and look at the induced map on $A_{\rho} \times B \rightarrow B$ defined by $f \circ a=f(V) a$, where $f$ belongs to $A_{\rho}$ and $a$ to $B$. The idea of considering the induced map is an adaptation of a technique developed by Domar in his study of function algebras over locally compact Abelian groups.

Using the continuity of the mapping $(f, a) \rightarrow f \circ a$, we may associate with each element $a$ in $B$ a closed subset $\Lambda(a)$ of the circumference of the unit circle defined as the hull of the ideal $I(a)=\left\{f \in A_{\rho}: f \circ a\right.$ $=0\}$. In addition, for each $f$ in $A_{\rho}$, let $\Lambda_{f}$ denote the closure of the support of $f$; then the following results are basic (cf. [3]).

LEMma 1. (a) $\Lambda(a)$ is void if and only if $a=0$.

(b) For any $f$ in $A_{\rho}$ and $a$ in $B, \Lambda(f \circ a) \subseteq \Lambda_{f} \cap \Lambda(a)$.

(c) If $f$ and $g$ belong to $A_{\rho}$ and $f \equiv g$ in some neighborhood of $\Lambda(a)$, then $f \circ a=g \circ a$.

The elements $\phi$ of the adjoint space $A_{\rho}^{*}$ may be identified with the space of sequences $\left\{\phi_{n}\right\}$ for which $\sup _{n}\left|\phi_{n}\right| / \rho_{n}<\infty$ by means of the representation $\phi(f)=\sum_{-\infty}^{\infty} f_{n} \phi_{n} ;$ furthermore $\|\phi\|=\sup _{n}\left|\phi_{n}\right| / \rho_{n}$. With each $\phi$ in $A_{\rho}^{*}$, there is associated a pair of functions $\Phi^{+}(z)$ $=\sum_{1}^{\infty} \phi z^{-n}, \Phi^{-}(z)=-\sum_{0}^{\infty} \phi_{-n} z^{n}$, where $\Phi^{+}$and $\Phi^{-}$are defined and analytic for $|z|>1$ and $|z|<1$, respectively. Using the pair $\left(\Phi^{+}, \Phi^{-}\right)$ we may define a closed set $\sigma(\phi)$ as the set of points $\lambda$ on the circumference of the unit circle for which the pair $\left(\Phi^{+}, \Phi^{-}\right)$do not continue each other across any arc containing the point $\lambda$ (cf. [2], [8]).

On the other hand we may construct another representation of $A_{\rho}$, this time as an operator over the Banach space $A_{\rho}^{*}$ in place of the space $B$. In this case the action of $f$ as an operator in $A_{\rho}^{*}$ will be defined by $(f \circ \phi)(g)=\phi(f g)$. With this representation the set $\Lambda(\phi)$ may be defined just as was done for the elements of $B$. In [3] it was shown 
that $\sigma(\phi)=\Lambda(\phi)$. Now for any $a$ in $B$ and $a^{*}$ in $B^{*}$, the sequence $\phi_{n}=a^{*}\left(V^{n-1} a\right)$ defines an element $\phi$ in $A_{\rho}^{*}$. Using the definition of $\sigma(\phi)$ we are led to the usual definition (cf. [5]) of the spectrum $\sigma(a)$ of an element $a$ in $B$ as the set of points $\lambda,|\lambda|=1$, such that for some $a^{*}$ in $B^{*}, \lambda$ belongs to the spectrum of $\left\{a^{*}\left(V^{n-1} a\right)\right\}$ as an element of $A_{\rho}^{*}$.

Theorem 1. For each $a$ in $B, \sigma(a)=\Lambda(a)$.

This result is established as follows. Let $a$ be a fixed element in $B$, and let $\phi_{a^{*}}$ denote the element in $A_{\rho}^{*}$ corresponding to the sequence $\left\{a^{*}\left(V^{n} a\right)\right\}$. Observe that since $(f g) \circ a=g \circ(f \circ a)$, we have

$$
\begin{aligned}
\bigcap\left\{I\left(\phi_{a^{*}}\right):\right. & \left.a^{*} \text { in } A_{\rho}^{*}\right\} \\
& =\left\{f \text { in } A_{\rho}: a^{*}[g \circ(f \circ a)]=0 \text { for all } g \text { in } A_{\rho} \text { and } a^{*} \text { in } A_{\rho}^{*}\right\} \\
& =\left\{f \text { in } A_{\rho}: g \circ(f \circ a)=0 \text { for all } g \text { in } A_{\rho}\right\}=I(a) .
\end{aligned}
$$

Hence $\Lambda\left(\phi_{a^{*}}\right) \subseteq \Lambda(a)$ for every $a^{*}$ in $B^{*}$; so that if $\Delta$ denotes the closure of the union of the sets $\Lambda\left(\phi_{a^{*}}\right)$ taken over all $a^{*}$ in $B^{*}$, then we have $\Delta \subseteq \Lambda(a)$. If $\Delta$ were a proper subset, then there would exist a point $t_{0}$ together with a closed neighborhood $N$ of $t_{0}$ such that $N \cap \Delta$ $=\varnothing$. But then $N \cap \Lambda\left(\phi_{a^{*}}\right)=\varnothing$ for every $a^{*}$ in $B^{*}$. Since $\Lambda\left(\phi_{a^{*}}\right)$ $=$ hull $I\left(\phi_{a^{*}}\right)$, we see that if $\Lambda_{f} \subset N$ and $f\left(t_{0}\right) \neq 0$, then $f$ belongs to $I\left(\phi_{a^{*}}\right)$ for all $a^{*}$ in $B^{*}$. But then $f$ belongs to $I(a)$. Since the point $t_{0}$ belongs to $\Lambda(a)$ we have $f\left(t_{0}\right)=0$, which is a contradiction. Hence, using the fact that $\Lambda\left(\phi_{a^{*}}\right)=\sigma\left(\phi_{a^{*}}\right)$, we have $\Lambda(a)=\Delta$ and $\Delta=$ closure of the union of the sets $\sigma\left(\phi_{a^{*}}\right)$ taken over all $a^{*}$ in $B^{*}$. On the other hand, it is easily seen that $\Delta \subseteq \sigma(a)$. Just as before, if $\Delta$ were a proper subset there would exist a point $t_{0}$ in $\sigma(a)$ and a closed neighborhood $N$ of $t_{0}$ such that $N \cap \sigma\left(\phi_{a^{*}}\right)=\varnothing$ for all $a^{*}$. But then $a^{*}\left[(t-V)^{-1} a\right]$ would be analytic in $N$ for every $a^{*}$ in $B^{*}$, contradicting the assumption that $t_{0}$ belongs to $\sigma(a)$.

In the following generalized approximation theorem, the role played by the range of the spectral projections in the case of a unitary operator is taken over by certain "spectral subspaces" defined in the following way. For each closed subset $\Delta$ of the circumference of the unit circle, set $M_{\Delta}=\{a$ in $B: \sigma(a) \subseteq \Delta\}$. Wermer [8] has shown that the linear manifold $\left\{a\right.$ in $\left.B: \Lambda_{a} \subseteq \Delta\right\}$ is closed; this, together with Theorem 1 , shows that $M_{\Delta}$ is indeed a subspace.

THeOREM 2. Given any $\epsilon>0$ there exists $a \delta>0$ such that for any $\lambda$ with $-\pi<\lambda<\pi$ and any element $a$ in $B$ which lies in the subpace $L(\lambda)=\{a$ in $B ; \sigma(a) \subseteq[\lambda-\delta, \lambda+\delta]\}$

$$
\left\|\left(V-e^{i \lambda}\right) a\right\| \leqq \epsilon\|a\| \text {. }
$$


Moreover, the space B is spanned by a finite collection of such manifolds.

The proof rests on the following lemma.

LEMмA 2. For any $\lambda$ in $[-\pi, \pi]$ and any $\epsilon>0$ there exists an $\eta>0$ and a function $f$ in $A_{\rho}$ such that

(i) $\|f\|=\sum_{-\infty}^{\infty} \rho_{n}\left|f_{n}\right|<\epsilon$, and

(ii) $f(\theta) \equiv e^{i \lambda}-e^{i \theta}$ for $|\theta-\lambda|<\eta$.

It suffices to find such an $f$ in the case where $\lambda=0$, since one has only to set $f_{\lambda}(\theta)=e^{i \lambda} f(\theta-\lambda)$, noting that $\left\|f_{\lambda}\right\|=\|f\|$, to obtain the general result. For any $\alpha>0$, set

$$
\begin{aligned}
f(\theta) & =1-e^{i \theta} \quad \text { for }|\theta| \leqq \alpha, \\
& =0 \quad \text { for }|\theta| \geqq 2 \alpha, \text { with } f \text { in } C^{\infty} .
\end{aligned}
$$

Then $\left|f_{0}\right| \leqq K_{0} \alpha$ and $\left|f_{n}\right| \leqq \alpha K_{l}|n|^{-l}$ for any integer $l$, where $K_{0}$ and $K_{l}$ depend on $f$. Taking $l \geqq q+2$ and recalling that $\rho_{n} \leqq M\left(1+|n|^{q}\right)$, we obtain $\|f\| \leqq \alpha K^{\prime}$ for some constant $K^{\prime}$. Letting $\eta=\epsilon / K^{\prime}$ completes the proof of the lemma.

Now if $\epsilon>0$ and $\lambda$ are given, we construct $f$ as in the above lemma and we set $\delta=\eta / 2$. Using Lemma 1 (c) we see that if $\sigma(a) \subseteq[\lambda-\delta$, $\lambda+\delta]$, then

$$
f \circ a=\left(e^{i \lambda}-e^{i \theta}\right) \circ a=\left(e^{i \lambda}-V\right) a .
$$

Hence

$$
\left\|\left(e^{i \lambda}-V\right) a\right\|=\|f \circ a\|<\epsilon\|a\| .
$$

For the second part, we cover the interval $[-\pi, \pi]$ with any finite collection $\left\{\Delta_{j}\right\}_{j=1}^{n}$ of overlapping intervals each having length equal to the $\delta$ found in the first part. Over this cover we construct a partition of unity $\left\{u_{j}\right\}_{j=1}^{n}$ with $u_{j} \in C^{\infty}$. Then for any element $a$ in $B$ we have $a=a_{1}+a_{2}+\cdots+a_{n}$, where $a_{j}=u_{j} \circ a$, and $\sigma\left(a_{j}\right) \subseteq \Lambda\left(u_{j}\right) \cap \sigma(a)$ $\subseteq \Delta_{j}$. Let $\lambda_{j}$ denote the center of $\Delta_{j}$ and $P_{j}(\theta)=e^{i \lambda_{j}}-e^{i \theta}$. Then, using the preceding lemma, there exists an $f^{(j)}$ in $A_{\rho}$ such that $\left\|f^{(j)}\right\|<\epsilon$ and $f^{(j)} \equiv P_{j}$ in a neighborhood of $\Delta_{j}$. Thus

$$
\left\|\left(e^{i \lambda_{j}}-V\right) a\right\|=\left\|f^{(j)} \circ a_{j}\right\| \leqq \epsilon\left\|a_{j}\right\| .
$$

Corollary. If $a \neq 0$, then $\sigma(a)=\left\{\lambda_{0}\right\}$ if and only if $V a=e^{i \lambda_{0}} a$.

\section{REFERENCES}

1. A. Beurling, Sur les intégrales de Fourier absolument convergentes et leur application a une transformation fonctionelle, 9th Congrès des Mathématiciens Scandinaves, 1938. 
2. - Sur les spectres des fonctions, Congrès sur L'Analyse Harmonique, Nancy, 1947.

3. Y. Domar, Harmonic analysis based on certain commutative Banach algebras, Acta Math. 96 (1956), 2-66.

4. N. Dunford, Spectral theory. II. Resolutions of the identity, Pacific J. Math. 2 (1952), 559-614.

5. - Spectral operators, Pacific J. Math. 4 (1954), 321-354.

6. G. Leaf, $A$ spectral theory for a class of linear operators, Pacific J. Math. 13 (1963), 141-155.

7. E. R. Lorch, The integral representation of weakly almost periodic transformations in reflexive vector spaces, Trans. Amer. Math. Soc. 49 (1941), 18-40. 622.

8. J. Wermer, The existence of invariant subspaces, Duke Math. J. 19 (1952), 615-

9. F. Wolf, Operators in Banach space which admit a generalized spectral decomposition, Proc. Akad. Wetensch. Ser. A 60 (1957), 302-311.

Argonne National Laboratory, Argonne, Illinois

\section{A NOTE ON NORMAL DILATIONS}

\section{ARNOLD LEBOW}

I. Introduction. Our purpose is to give certain sufficient conditions that a normal dilation of an operator be an extension from a reducing subspace. The first result of this kind, that we know of, is due to T. Andô [1] who considered compact normal dilations. In this note we use only assumptions about the nature of the spectrum; nevertheless, we are able to recover Andô's theorem.

Let $A$ be an operator on a Hilbert space $\Re$. Let $P$ be the orthogonal projection of $\nVdash$ onto a subspace $\Re$. Let $T$ denote the restriction of $P A P$ to $\mathcal{F C}$. The operator $T$ is called a compression of $A$ and $A$ is called a dilation of $T$. If $T^{n}$ is the compression of $A^{n}(n=01,2,3, \cdots)$ then $T$ is called a strong compression and $A$ a strong dilation. Let $X$ be a compact subset of the plane containing $\sigma(A)$ and $\sigma(T)$, the spectra of $A$ and $T$. The operator $A$ is said to be an $X$-dilation of $T$ if, for every rational function $r(\cdot)$ which is analytic on $X$, the operator $r(A)$ is a dilation of $r(T)$. These definitions were introduced by Halmos. Some other writers use "dilation" to mean what we call strong dilation. Sz-Nagy uses "projection" for compression. When $T$ is a strong compression of $A$ Andô calls $\mathfrak{H}$ a "semi-invariant" subspace of $A$.

These notions are related to the more familiar concepts of invariant subspace and reducing subspace as follows. If $\mathcal{T C}$ is an invariant sub-

Presented to the Society, January 27, 1965 under the title On a theorem of Andô; received by the editors September 21, 1964. 